\title{
FUNCTIONS COMMUTING WITH AN ARBITRARY FIXED BIJECTION
}

\author{
ANDRZEJ MACH
}

\begin{abstract}
In this note a characterization of all bijective functions commuting with a fixed bijection $\varphi: X \rightarrow X$, where $X$ is an arbitrary nonempty set, is given.
\end{abstract}

\section{INTRODUCTION}

One can find commuting functions in many papers devoted to functional equations or iteration theory, see for example [1], [2], [3], [5], [6]. Therefore, the problem of describing commuting functions with a given one seems to be interesting. The aim of this paper is a solution of the commutativity problem for all bijective functions. We present the form of commuting functions separately for closed and open orbits (see [4], page 15) of a given bijection $\varphi: X \rightarrow X$.

\section{Functions commuting With a solution of Babbage functional EQUATION}

1.1. Notations, definitions, lemma. Let us start with introducing some notations and definitions. Let $X$ be an arbitrary nonempty set. Let $\varphi: X \rightarrow$ $X$ satisfy the Babbage equation (described in [4], page 288), this means

$$
\varphi^{n}(x)=x, \quad n \geq 2,
$$

(by $\varphi^{n}$ we denote $n$-th iteration).

Let us define

$$
D:=\{m: m \text { divides } n\}
$$

and

$$
X^{(m)}:=\left\{x \in X: \varphi^{m}(x)=x \text { and } \varphi^{k}(x) \neq x \text {, for every } 1 \leq k<m\right\} .
$$

2010 Mathematics Subject Classification. Primary 39B12; Secondary 26A18.

Key words and phrases. Bijective function, commuting function, iteration, closed and open orbits, Babbage functional equation. 
Lemma 1.1 ([5] or [7]). The sets $\left\{X^{(m)}: m \in D\right\}$ are pairwise disjoint and the equality

$$
\bigcup_{m \in D} X^{(m)}=X
$$

holds.

For convenience of the reader we quote the proof from the paper [7].

Proof. It is evident that the sets $\left\{X^{(m)}\right\}_{m \in D}$ are pairwise disjoint and $\bigcup_{m \in D}$ $X^{(m)} \subset X$. To prove the reverse inclusion, we reason as follows: if $x \in X$ and $p \in\{1,2, \ldots, n\}$ is the minimal number such that $\varphi^{p}(x)=x$ then, evidently, for $t \in \mathbf{N} \cup\{0\}$ satisfying the inequalities

$$
\frac{n-p}{p} \leq t \leq \frac{n-1}{p}
$$

we have

$$
1 \leq n-t p \leq p \quad \text { and } \quad \varphi^{t p}(x)=x .
$$

Therefore

$$
\varphi^{n-t p}(x)=\varphi^{n-t p}\left(\varphi^{t p}(x)\right)=\varphi^{n}(x)=x .
$$

Hence $n-t p=p$, whence $p(t+1)=n$.

For every $m \in D$, by $S_{m}$, we denote an arbitrary selection of the family of orbits

$$
\left\{\left\{x, \varphi(x), \varphi^{2}(x), \ldots, \varphi^{m-1}(x)\right\}: x \in X^{(m)}\right\} .
$$

\subsection{Main constructions for a solution of the Babbage equation.}

Proposition 1.1. Let us suppose that $m \in D$ is fixed, $X=X^{(m)}$ and $S=S_{m}$. Let $g^{*}: S \rightarrow S$ be an arbitrary bijection. Moreover, let $k: X \rightarrow$ $\{0,1, \ldots, m-1\}$ be defined as follows

$$
k(x):=k \quad \Leftrightarrow \quad \varphi^{k}(x) \in S .
$$

The function $g: X \rightarrow X$ defined by the formula

$$
g(x):=\left[\varphi^{m-k(x)} \circ g^{*} \circ \varphi^{k(x)}\right](x)
$$

commutes with the function $\varphi$.

Proof. Firstly, let us remark that (1.2) implies $k(\varphi(x))=k(x)-1$ if $k(x) \neq 0$ and $k(\varphi(x))=m-1$ if $k(x)=0$. We have for $k(x) \neq 0$

$$
\begin{aligned}
& g(\varphi(x))=\left[\varphi^{m-k(\varphi(x))} \circ g^{*} \circ \varphi^{k(\varphi(x))}\right](\varphi(x))=\left[\varphi^{m-k(\varphi(x))} \circ g^{*} \circ \varphi^{k(\varphi(x))+1}\right](x) \\
& =\left[\varphi^{m-k(x)+1} \circ g^{*} \circ \varphi^{k(x)-1+1}\right](x)=\varphi\left(\left[\varphi^{m-k(x)} \circ g^{*} \circ \varphi^{k(x)}\right](x)\right)=\varphi(g(x)) .
\end{aligned}
$$


Similarly, for $k(x)=0$ we have

$$
\begin{aligned}
& g(\varphi(x))=\left[\varphi^{m-k(\varphi(x))} \circ g^{*} \circ \varphi^{k(\varphi(x))}\right](\varphi(x))=\left[\varphi^{m-k(\varphi(x))} \circ g^{*} \circ \varphi^{k(\varphi(x))+1}\right](x) \\
& =\left[\varphi^{m-m+1} \circ g^{*} \circ \varphi^{m-1+1}\right](x)=\varphi\left(\left[\varphi^{m-k(x)} \circ g^{*} \circ \varphi^{k(x)}\right](x)\right)=\varphi(g(x)) .
\end{aligned}
$$

Remark 1.2. Let us remark that in the case $m=1$ we have $\varphi=i d_{X}$, $S=X$ and by the formula (1.3) we get $g=g^{*}: X \rightarrow X$, i.e. every bijection commutes with the identity function.

Proposition 1.2. The function g given by the equality $g=\bigcup_{m \in D} g_{m}$, where $g_{m}: X^{(m)} \rightarrow X^{(m)}$ has the form (1.3) commutes with the function $\varphi: X \rightarrow$ $X$ such that eq. (1.1) holds.

The proof results immediately from $\varphi\left(X^{(m)}\right)=X^{(m)}$, Lemma 1.1 and Proposition 1.1.

Example 1.3. Using Propositions 1.1 and 1.2 one can easily obtain the following: if $\left.g^{*}:\right] \frac{1}{2}, \infty[\rightarrow] \frac{1}{2}, \infty[$ is an arbitrary bijection then the function

$$
g(x)= \begin{cases}g^{*}(x) & \text { for } \quad x>\frac{1}{2} \\ \frac{1}{2} & \text { for } \quad x=\frac{1}{2} \\ 1-g^{*}(1-x) & \text { for } x<\frac{1}{2}\end{cases}
$$

commutes with the function $\varphi(x)=1-x$, for $x \in \mathbb{R}$. Particularly, the functions

$$
g(x)=\left\{\begin{array}{lll}
\frac{1}{2}+\frac{2}{2 x-1} & \text { for } x>\frac{1}{2}, \\
\frac{1}{2} & \text { for } x=\frac{1}{2}, \\
\frac{1}{2}-\frac{2}{1-2 x} & \text { for } x<\frac{1}{2},
\end{array} \quad g(x)= \begin{cases}\frac{1}{2}+\ln \left(x+\frac{1}{2}\right) & \text { for } x>\frac{1}{2} \\
\frac{1}{2} & \text { for } x=\frac{1}{2} \\
\frac{1}{2}-\ln \left(\frac{3}{2}-x\right) & \text { for } x<\frac{1}{2}\end{cases}\right.
$$

commute with the function $\varphi(x)=1-x$, for $x \in \mathbb{R}$.

Example 1.4. Let $X=\mathbb{R} \backslash\{0\}$ and $\varphi(x):=\frac{1}{x}, x \in X$. Let $g_{1}^{*}:\{-1,1\} \rightarrow$ $\{-1,1\}$ be an arbitrary bijection. Let us consider the following cases:

a) $S:=]-1,0[\cup] 0,1[$, or

b) $S:=]-1,0[\cup] 1,+\infty[$, or

c) $S:=]-\infty,-1[\cup] 0,1[$, or

d) $S:=]-\infty,-1[\cup] 1,+\infty[$.

Let $g_{2}^{*}: S \rightarrow S$ be an arbitrary bijection. Using Propositions 1.1 and 1.2 the function

$$
g(x)=\left\{\begin{array}{lll}
g_{1}^{*}(x) & \text { for } \quad x \in\{-1,1\} \\
g_{2}^{*}(x) & \text { for } \quad x \in S \\
\frac{1}{g_{2}^{*}\left(\frac{1}{x}\right)} & \text { for } \quad x \in X \backslash S
\end{array}\right.
$$


commutes with the function $\varphi(x)$. Particularly, the functions $g(x)=x^{n}$, $g(x)=\frac{1}{x^{n}}$

$$
\begin{aligned}
& g(x)= \begin{cases}1 & \text { for } x=-1, \\
-1 & \text { for } x=1, \\
\sin \frac{\pi}{2} x & \text { for } x \in]-1,0[\cup] 0,1[, \\
\frac{1}{\sin \frac{\pi}{2 x}} & \text { for } x \in]-\infty,-1[\cup] 1,+\infty[,\end{cases} \\
& g(x)= \begin{cases}i d_{\{-1,1\}} & \text { for } x \in\{-1,1\}, \\
e^{x+1} & \text { for } x \in]-\infty,-1[, \\
\ln x-1 & \text { for } x \in] 0,1[, \\
\frac{1}{e^{\frac{1}{x}+1}} & \text { for } x \in]-1,0[, \\
\frac{1}{\ln \frac{1}{x}-1} & \text { for } x \in] 1,+\infty[,\end{cases}
\end{aligned}
$$

commute with the function $\varphi(x)=\frac{1}{x}$, for $x \in \mathbb{R} \backslash\{0\}$.

Example 1.5. Let $A$ and $Y$ be arbitrary nonempty sets and $\operatorname{card} Y \geq 12$. Let us write $I_{p}:=\{0,1, \ldots, p\}$, for $p=0,1, \ldots$. Let $X:=A \cup B$, where $B:=\left\{\sigma_{i}^{j} \in Y: i \in I_{3}, j \in I_{4}\right\}$ and $\operatorname{cardB}=12$. Let $\varphi \in X^{X}$ be defined by

$$
\varphi(x):= \begin{cases}x, & \text { if } x \in A, \\ \sigma_{(i+1)_{4}}^{j}, & \text { if } x=\sigma_{i}^{j}, i \in I_{3}, j \in I_{4}\end{cases}
$$

where $(i+1)_{4}$ denotes the remainder of division of $i+1$ by 4 . Then $\varphi^{4}(x)=$ $x, x \in X$. In this case we have $n=4, X^{(1)}=A, X^{(2)}=\emptyset, X^{(4)}=B$. Let $g_{1}^{*}: A \rightarrow A$ be an arbitrary bijection. Let $S:=\left\{\sigma_{0}^{j}: j \in I_{4}\right\}$. Take the bijection $g_{2}^{*}: S \rightarrow S$ defined as follows: $g_{2}^{*}\left(\sigma_{0}^{j}\right):=\sigma_{0}^{(j+1)_{5}}$. According to Propositions 1.1 and 1.2 the function $g: X \rightarrow X$ defined by the formula

$$
g(x)= \begin{cases}g_{1}^{*}(x) & \text { for } \quad x \in A, \\ \sigma_{(i)_{4}}^{(j+1)_{5}} & \text { for } \quad x=\sigma_{i}^{j} \in B,\end{cases}
$$

commutes with $\varphi$.

We define the relation in $X$ by

$$
x \sim y \quad \Leftrightarrow \quad \varphi^{p}(x)=y, \text { for a } p \in\{0,1, \ldots, m-1\} .
$$

One can easily observe that it is an equivalence relation.

Let $S$ be a selection of the family of orbits $\left\{\left\{x, \varphi(x), \varphi^{2}(x), \ldots, \varphi^{m-1}(x)\right\}\right.$ : $x \in X\}$. Then there exists a unique function $h_{S}: X \rightarrow S$ such that $h_{S}(x) \sim$ $x$. Note that $h_{S}(\varphi(x))=h_{S}(x)$.

Theorem 1.6. Let $X$ be an arbitrary nonempty set and $n \geq 2$. Let $\varphi$ : $X \rightarrow X$ be a solution of the Babbage equation (1.1) and suppose that $X=$ 
$X^{(m)}$ for a fixed $m \in D$. The bijection $g: X \rightarrow X$ commutes with the solution $\varphi$ if and only if there exist a selection $S$ of the family of orbits $\left\{\left\{x, \varphi(x), \varphi^{2}(x), \ldots, \varphi^{m-1}(x)\right\}: x \in X\right\}$, a bijection $g^{*}: S \rightarrow S$ and $a$ function $p: S \rightarrow\{0,1, \ldots, m-1\}$ such that $g$ has the form

$$
g(x):=\left[\varphi^{m-k(x)+p\left(h_{S}(x)\right)} \circ g^{*} \circ \varphi^{k(x)}\right](x)
$$

where $k: X \rightarrow\{0,1, \ldots, m-1\}$ is defined by (1.2).

Proof of the "if" part. Evidently, we have if $x \sim h_{S}(x), h_{S}(\varphi(x))=h_{S}(x)$ and further reasoning is similar as in Proposition 1.1. We have for $k(x) \neq 0$

$$
\begin{aligned}
g(\varphi(x)) & =\left[\varphi^{m-k(\varphi(x))+p\left(h_{S}(\varphi(x))\right)} \circ g^{*} \circ \varphi^{k(\varphi(x))}\right](\varphi(x)) \\
& =\left[\varphi^{m-k(\varphi(x))+p\left(h_{S}(\varphi(x))\right)} \circ g^{*} \circ \varphi^{k(\varphi(x))+1}\right](x) \\
& =\left[\varphi^{m-k(x)+1+p\left(h_{S}(x)\right)} \circ g^{*} \circ \varphi^{k(x)-1+1}\right](x) \\
& =\varphi\left(\left[\varphi^{m-k(x)+p\left(h_{S}(x)\right)} \circ g^{*} \circ \varphi^{k(x)}\right](x)\right)=\varphi(g(x)) .
\end{aligned}
$$

Similarly, for $k(x)=0$ we have

$$
\begin{aligned}
g(\varphi(x)) & =\left[\varphi^{m-k(\varphi(x))+p\left(h_{S}(\varphi(x))\right)} \circ g^{*} \circ \varphi^{k(\varphi(x))}\right](\varphi(x)) \\
& =\left[\varphi^{m-k(\varphi(x))+p\left(h_{S}(\varphi(x))\right)} \circ g^{*} \circ \varphi^{k(\varphi(x))+1}\right](x) \\
& =\left[\varphi^{m-m+1+p\left(h_{S}(x)\right)} \circ g^{*} \circ \varphi^{m-1+1}\right](x) \\
& =\varphi\left(\left[\varphi^{m-k(x)+p\left(h_{S}(x)\right)} \circ g^{*} \circ \varphi^{k(x)}\right](x)\right)=\varphi(g(x)) .
\end{aligned}
$$

Proof of the "only if" part. Let $g: X \rightarrow X$ be a bijection commuting with $\varphi$. Take an arbitrary selection $S$ of the family of orbits $\left\{\left\{x, \varphi(x), \varphi^{2}(x), \ldots\right.\right.$, $\left.\left.\varphi^{m-1}(x)\right\}: x \in X\right\}$. One can observe that every function $g^{*}: S \rightarrow S$ defined in such a way that $g^{*}(s)=t$ for a $t$ if and only if $g(s) \sim t$, is a bijection. Let $x \in X$ and put $s:=h_{S}(x)$. There exists $p(s) \in\{0,1, \ldots, m-1\}$ such that $\varphi^{p(s)}\left(g^{*}(s)\right)=g(s)$. Moreover we have

$$
\varphi^{k(x)}(x)=s, \varphi^{m-k(x)}(s)=x .
$$

From the above

$$
\begin{gathered}
{\left[\varphi^{m-k(x)+p(s)} \circ g^{*} \circ \varphi^{k(x)}\right](x)=\varphi^{m-k(x)+p(s)}\left[g^{*}(s)\right]=\varphi^{m-k(x)+p(s)}(t)} \\
\quad=\varphi^{m-k(x)}(g(s))=g\left(\varphi^{m-k(x)}(s)\right)=g(x) .
\end{gathered}
$$


Theorem 1.7. Let $X$ be an arbitrary nonempty set and $n \geq 2$. Let $\varphi: X \rightarrow$ $X$ satisfy the Babbage equation (1.1). The bijection $g: X \rightarrow X$ commutes with the solution $\varphi$ if and only if $g=\bigcup_{m \in D} g_{m}$, where $g_{m}: X^{(m)} \rightarrow X^{(m)}$ have the form (1.6).

The proof results immediately from $\varphi\left(X^{(m)}\right)=X^{(m)}$, Lemma 1.1 and Theorem 1.6.

Example 1.8. Let $X=B$, where $B$ is defined as in Example 1.5. Let $\varphi$ be defined by $\varphi\left(\sigma_{i}^{j}\right)=\sigma_{(i+1)_{4}}^{j}$, for $i \in I_{3}, j \in I_{4}$. Let $S$ be the same as in Example 1.5, so $S:=\left\{\sigma_{0}^{j}: j \in I_{4}\right\}$. Take the bijection $g^{*}: S \rightarrow S$ defined as follows: $g^{*}\left(\sigma_{0}^{j}\right):=\sigma_{0}^{(j+2)_{5}}$. Define the function $p: S \rightarrow\{0,1,2,3\}$ by $p\left(\sigma_{0}^{j}\right)=(j)_{4}$. By the formula (1.6) we get the function $g\left(\sigma_{i}^{j}\right)=\sigma_{(j+i)_{4}}^{(j+2)_{5}}$. One can easily verify that $g$ commutes with $\varphi$.

\section{THE CASE OF OPEN ORBITS}

Let $X$ be an arbitrary nonempty set. Let $\varphi: X \rightarrow X$ be a bijection such that

$$
\varphi^{n}(x) \neq x, \quad \forall n \geq 1 .
$$

In this case all orbits are as follows:

$$
\left\{\ldots, \varphi^{-3}(x), \varphi^{-2}(x), \varphi^{-1}(x), x, \varphi(x), \varphi^{2}(x), \varphi^{3}(x), \varphi^{4}(x), \ldots\right\} .
$$

Now, we define the equivalence relation in $X$ by

$$
x \sim y \quad \Leftrightarrow \quad \varphi^{p}(x)=y, \text { for a } p \in \mathbb{Z} .
$$

Let $S$ be a selection of the family of orbits

$$
\left\{\left\{\ldots, \varphi^{-3}(x), \varphi^{-2}(x), \varphi^{-1}(x), x, \varphi(x), \varphi^{2}(x), \varphi^{3}(x), \varphi^{4}(x), \ldots\right\}: x \in X\right\} .
$$

Then there exists a unique function $h_{S}: X \rightarrow S$ such that $h_{S}(x) \sim x$. Note that $h_{S}(\varphi(x))=h_{S}(x)$.

Theorem 2.1. Let $X$ be an arbitrary nonempty set. Let $\varphi: X \rightarrow X$ be a bijective function satisfying (2.1). The bijection $g: X \rightarrow X$ commutes with the function $\varphi$ if and only if there exist a selection $S$ of the family of orbits

$$
\left\{\left\{\ldots, \varphi^{-3}(x), \varphi^{-2}(x), \varphi^{-1}(x), x, \varphi(x), \varphi^{2}(x), \varphi^{3}(x), \varphi^{4}(x), \ldots\right\}: x \in X\right\},
$$

a bijection $g^{*}: S \rightarrow S$ and a function $p: S \rightarrow \mathbb{Z}$ such that $g$ has the form

$$
g(x):=\left[\varphi^{-k(x)+p\left(h_{S}(x)\right)} \circ g^{*} \circ \varphi^{k(x)}\right](x)
$$

where $k: X \rightarrow \mathbb{Z}$ is defined by (1.2). 
Proof of the "if" part. Evidently, we have if $x \sim h_{S}(x), h_{S}(\varphi(x))=h_{S}(x)$ and further reasoning is similar as in Proposition 1.1 and in Theorem 1.6. Indeed, we have $k(\varphi(x))=k(x)-1$ for all $x \in X$, so

$$
\begin{aligned}
& g(\varphi(x))=\left[\varphi^{-k(\varphi(x))+p\left(h_{S}(\varphi(x))\right)} \circ g^{*} \circ \varphi^{k(\varphi(x))}\right](\varphi(x)) \\
& =\left[\varphi^{-k(\varphi(x))+p\left(h_{S}(\varphi(x))\right)} \circ g^{*} \circ \varphi^{k(\varphi(x))+1}\right](x) \\
& =\left[\varphi^{-k(x)+1+p\left(h_{S}(x)\right)} \circ g^{*} \circ \varphi^{k(x)-1+1}\right](x) \\
& =\varphi\left(\left[\varphi^{-k(x)+p\left(h_{S}(x)\right)} \circ g^{*} \circ \varphi^{k(x)}\right](x)\right)=\varphi(g(x)) .
\end{aligned}
$$

Proof of the "only if" part. Let $g: X \rightarrow X$ be a bijection commuting with $\varphi$. Take an arbitrary selection $S$ of the family of orbits

$$
\left\{\left\{\ldots, \varphi^{-3}(x), \varphi^{-2}(x), \varphi^{-1}(x), x, \varphi(x), \varphi^{2}(x), \varphi^{3}(x), \varphi^{4}(x), \ldots\right\}: x \in X\right\} .
$$

One can observe that every function $g^{*}: S \rightarrow S$ defined in such a way that $g^{*}(s)=t$ for a $t$ if and only if $g(s) \sim t$, is a bijection. Let $x \in X$ and put $s:=h_{S}(x)$. There exists $p(s) \in \mathbb{Z}$ such that $\varphi^{p(s)}\left(g^{*}(s)\right)=g(s)$. Moreover we have

$$
\varphi^{k(x)}(x)=s, \varphi^{-k(x)}(s)=x .
$$

From the above

$$
\begin{gathered}
{\left[\varphi^{-k(x)+p(s)} \circ g^{*} \circ \varphi^{k(x)}\right](x)=\varphi^{-k(x)+p(s)}\left[g^{*}(s)\right]=\varphi^{-k(x)+p(s)}(t)} \\
=\varphi^{-k(x)}(g(s))=g\left(\varphi^{-k(x)}(s)\right)=g(x) .
\end{gathered}
$$

\section{Final Remarks, examples and problems}

Remark 3.1. The results of this paper can be useful in finding all functions commuting with a given one. Namely, by Theorem 1.7, we have a ready to use description of commuting functions for all closed orbits of the given bijective function. Similarly, in Theorem 2.1, we have a ready to use construction of commuting functions for all open orbits of the given bijective function. Since the union of the closed orbits $X_{I}$ and the union of open orbits $X_{I I}$ are disjoint and $\varphi\left(X_{I}\right)=X_{I}, \varphi\left(X_{I I}\right)=X_{I I}$, then the presented results are complete for the characterization of all commutative functions 
with a given bijection $\varphi$. More precisely, the domain $X$ of the bijection $\varphi$ can be decomposed onto disjoint sets as follows

$$
X=\bigcup_{n=1}^{\infty} X^{(n)} \cup X^{(\infty)},
$$

where $X^{(n)}=\left\{x \in X: \varphi^{n}(x)=x\right.$ and $\varphi^{k}(x) \neq x$ for $\left.0<k<n\right\}$ and $X^{(\infty)}=\left\{x \in X: \varphi^{n}(x) \neq x\right.$ for $\left.n \geq 1\right\}$. Theorems 1.6, 1.7 and Remark 1.2 describe the form of commuting function $g$ on the sets $X^{(n)}, n \geq 1$ and Theorem 2.1 describes the form of $g$ on the set $X^{(\infty)}$.

Example 3.2. Let us consider the bijection $\varphi: \mathbb{R}_{+} \rightarrow \mathbb{R}_{+}$given by the formula $\varphi(x)=3 x$. Using the presented theorems we can construct all functions $g$ commuting with $\varphi$. We have $\mathbb{R}_{+}=\mathbb{R}_{+}^{(1)} \cup \mathbb{R}_{+}^{(\infty)}$, where $\mathbb{R}_{+}^{(1)}=\{0\}$ and $\mathbb{R}_{+}^{(\infty)}=\mathbb{R}_{+} \backslash\{0\}$. Therefore $g(0)=0$ and to define $g$ on the set $\mathbb{R}_{+}^{(\infty)}$ we use Theorem 2.1. The interval [1,3[ forms a selection of the family of orbits $\left\{\left\{\ldots, \varphi^{-3}(x), \varphi^{-2}(x), \varphi^{-1}(x), x, \varphi(x), \varphi^{2}(x), \varphi^{3}(x), \varphi^{4}(x), \ldots\right\}: x \in \mathbb{R}_{+}^{(\infty)}\right\}$.

Moreover

$$
\mathbb{R}_{+}^{(\infty)}=\bigcup_{l \in \mathbb{Z}}\left[3^{l}, 3^{l+1}[\right.
$$

and for $x \in\left[3^{l}, 3^{l+1}[, l \in \mathbb{Z}\right.$ - according with (1.2) - we have $k(x)=-l$. The formula below gives a family of functions which commute with $\varphi$.

$$
g(x)= \begin{cases}0 & \text { for } \quad x=0, \\ 3^{p(s)+l} \cdot g^{*}\left(3^{-l} x\right) & \text { for } \quad x \in\left[3^{l}, 3^{l+1}[\text { and } l \in \mathbb{Z},\right.\end{cases}
$$

where $g^{*}:[1,3[\rightarrow[1,3[$ is an arbitrary bijection and $p:[1,3[\rightarrow \mathbb{Z}$ is an arbitrary function. Taking the bijection $g^{*}:[1,3[\rightarrow[1,3[$ defined by the formula $g^{*}(x)=1+2 \sin \frac{\pi}{4}(x-1)$ and taking the function $p(s)=7$, for $s \in[1,3$ [, we obtain from the above the form of the commuting function $g$ as follows

$$
g(x)= \begin{cases}0 & \text { for } \quad x=0, \\ 3^{7+l} \cdot\left[1+2 \sin \frac{\pi}{4}\left(3^{-l} x-1\right)\right] & \text { for } \quad x \in\left[3^{l}, 3^{l+1}[\text { and } l \in \mathbb{Z} .\right.\end{cases}
$$

Example 3.3. For the bijection $\varphi: \mathbb{R}_{+} \rightarrow \mathbb{R}_{+}$given by the formula $\varphi(x)=$ $x^{3}$ we have $\mathbb{R}_{+}=\mathbb{R}_{+}^{(1)} \cup \mathbb{R}_{+}^{(\infty)}$, where $\mathbb{R}_{+}^{(1)}=\{0,1\}$ and $\mathbb{R}_{+}^{(\infty)}=\mathbb{R}_{+} \backslash\{0,1\}$. Let $g_{1}^{*}:\{0,1\} \rightarrow\{0,1\}$ be an arbitrary bijection. Let $g_{2}^{*}:\left[\frac{1}{8}, \frac{1}{2}[\cup[2,8[\rightarrow\right.$ $\left[\frac{1}{8}, \frac{1}{2}\left[\cup\left[2,8\left[\right.\right.\right.\right.$ be an arbitrary bijection and $p:\left[\frac{1}{8}, \frac{1}{2}[\cup[2,8[\rightarrow \mathbb{Z}\right.$ be an arbitrary function. We have

$$
\mathbb{R}_{+}^{(\infty)}=\bigcup_{l \in \mathbb{Z}}\left[2^{-3^{l+1}}, 2^{-3^{l}}\left[\cup \bigcup _ { l \in \mathbb { Z } } \left[2^{3^{l}}, 2^{3^{l+1}}[\right.\right.\right.
$$


and $k(x)=-l$, for $x \in\left[2^{-3^{l+1}}, 2^{-3^{l}}\left[\cup\left[2^{3^{l}}, 2^{3^{l+1}}[, l \in \mathbb{Z}\right.\right.\right.$. The formula below gives a family of functions which commute with $\varphi$.

$$
g(x)= \begin{cases}g_{1}^{*}(x) & \text { for } \quad x \in\{0,1\}, \\ {\left[g_{2}^{*}\left(x^{3^{-l}}\right)\right]^{3^{p(s)+l}}} & \text { for } \quad x \in\left[2^{-3^{l+1}}, 2^{-3^{l}}\left[\cup \left[2^{3^{l}}, 2^{3^{l+1}}[\text { and } l \in \mathbb{Z} .\right.\right.\right.\end{cases}
$$

Example 3.4. For the bijection $\varphi: \mathbb{R} \rightarrow \mathbb{R}$ given by the formula $\varphi(x)=-x^{3}$ we have $\mathbb{R}=\mathbb{R}^{(1)} \cup \mathbb{R}^{(2)} \cup \mathbb{R}^{(\infty)}$, where $\mathbb{R}^{(1)}=\{0\}, \mathbb{R}^{(2)}=\{-1,1\}$ and $\mathbb{R}^{(\infty)}=\mathbb{R} \backslash\{-1,0,1\}$. Theorems 1.6, 1.7 and Remark 1.2 describe the form of the commuting function $g$ on the sets $\mathbb{R}^{(1)}, \mathbb{R}^{(2)}$ and Theorem 2.1 describes the form of $g$ on the set $\mathbb{R}^{(\infty)}$. Determining the parameters as in the previous examples we can obtain all functions commuting with the given function $\varphi$.

Problem 3.5. Characterize all functions commuting with a given finction $f: X \rightarrow X$ (not necessarily bijective), where $X$ is an arbitrary nonempty set, particularly for $f: \mathbb{R} \rightarrow \mathbb{R}$.

Acknowledgements. I wish to thank to anonymous referee for all valuable remarks.

\section{REFERENCES}

[1] D. Krassowska and M. C. Zdun, On limit sets of mixed iterates of commuting mappings, Aequationes Math., 78 (2009), 283-295.

[2] D. Krassowska and M. C. Zdun, On the embeddability of commuting continuous injections in iteration semigroups, Publicationes Math., 75 (2009), 179-190.

[3] D. Krassowska, Iteration groups and commuting functions in $\mathbb{R}^{n}$, Grazer Math. Berichte, 351 (2007), 100-109.

[4] M. Kuczma, Functional Equations in a Single Variable, Monografie Mat. 46, Polish Scientific Publishers (PWN), Warszawa 1968.

[5] A. Mach, The translation equation on certain n-groups, Aequationes Math., 47 (1994), $11-30$.

[6] A. Mach, The construction of the solutions of the generalized translation equation, Aequationes Math., 64 (2002), 1-23.

[7] A. Mach, On some functional equations involving Babbage equation, Result. Math., 51 (2007), 97-106.

(Received: November 5, 2012)

(Revised: February 20, 2013)
Faculty of Economics

Department of Social and Technical Science University of Business and Enterprise

ul. Akademicka 12

27-400 Ostrowiec Świetokrzyski

Poland

amach@wsbip.edu.pl

or

Faculty of Engineering and Economics 
The State Higher School of Vocational

Education

Narutowicza 9

06-400 Ciechanów

Poland

andrzej.mach@pwszciechanow.edu.pl 